\title{
Research on Dynamic and Mechanical Properties of Magneto- active Elastomers with High Permeability Magnetic Filling Agent at Complex Magneto-Temperature Exposure
}

\author{
Maria Vasilyeva ${ }^{1, *}$, Dmitry Nagornov ${ }^{1}$ and Grigory Orlov ${ }^{1}$ \\ 1 Saint-Petersburg mining university, 199106, Saint-Petersburg, Russia; saturn.sun@mail.ru \\ * Correspondence: saturn.sun@mail.ru
}

\begin{abstract}
The paper describes the research findings on dynamic and mechanical properties of composite elastomers with high permeability magnetic filling agent capable of rapidly and reversibly changing its properties when exposed to an external magnetic field, which makes it a perspective material for application in a wide range of engineering areas. The research has analyzed the trends in the strength properties of the materials obtained through the use of filling agents of different fineness, content, and structural organization in the final polymer, under different conditions. This allowed to obtain the correlations between the influence of the filler's relative fineness on the dynamic and mechanical properties of composite polymers, and to estimate the trends in the parameters describing the material strength under the complex magnetic and thermal influence. The results obtained by the authors allowed to ground the composition and structural organization of the final material with the best set of dynamic and mechanical properties.
\end{abstract}

Keywords: composite polymers, magnetoactive elastomers, dynamic and mechanical properties, concentration, anisotropy, fineness

\section{Introduction}

Magnetoactive elastomers (MAEs) represent a new class of materials with magnetic fields that can change their mechanical properties in a controlled manner. The filler particles in this material have a strong dipole magnetic interaction, such interaction depends on the saturation magnetic moment value determining the use of materials with high iron or cobalt content as a filling agent [1-3]. Considering the positive properties of this material, in recent years there have been a lot of research to its application in the aerospace, automotive, construction, and medicine industries [4-6].

The nature-like magnetic pump developed at the St. Petersburg Mining University (St. Petersburg, Russia) is one of the promising applications of magnetoactive elastomers that implements the principle of peristaltic transfer through displacement of local deformation waves of the channel chamber produced of magnetoactive elastomer generated under the influence of an electromagnetic field $[7,8]$. To a large extent, such pump units do not have disadvantages typical for traditional large units, because their performance depends on the electromagnetic field source properties (speed of local deformation wave motion, power) thereby eliminating the pressure losses depending on the pumped medium properties [9].

In order to assess the pipeline material ability to provide the required head-capacity curves of the pumping unit, it was important to study the effect of MAE composition, filling agent percentage, and the impact of its structure degree of order on its dynamic and mechanical properties in the application of external magnetic and temperature fields. 


\section{Materials and Methods}

\subsection{Elastomer material}

In course of the MAEs production, vinyl- or rubber-based polymers are used as a matrix [10 - 12], and microscopic particles of a magnetoactive filling agent are used as a filler $[13,14]$.

For the purposes of this research, a two-component silicone compound UNISIL 9115 has been used, the compound had the following specifications: viscosity - 15,000 MPa. sec, Shore hardness - $15 \mathrm{~A}$, elasticity - 500\%, tensile strength - $3.5 \mathrm{MPa}$, split sample tear resistance - $12 \mathrm{kN} / \mathrm{m}$, linear shrinkage - $1 \%$.

The two following types of high permeability magnetic materials were used as filling agents:

- atomized powdered iron (complies with GOST 9849-86): bulk density - $2.8 \mathrm{~g} / \mathrm{cm}^{3}$, grain-size composition basic fraction - $150 \mu \mathrm{m}$. The relative magnetic permeability $5.0 \cdot 103 \mu / \mu_{0}$.

- carbonyl iron (complies with GOST 13610-79): bulk density $2.0 \mathrm{~g} / \mathrm{cm}^{3}$, grain-size composition basic fraction - $5 \mu \mathrm{m}$. The relative magnetic permeability - $15 \mu / \mu_{0}$.

The compound was mixed by ferromagnetic particles to yield MAEs with the filling content of each type at 50,60, and 70\%, respectively. Further, the catalyst was added in a 100:3 ratio and then the mixture was degassed. Then, based on the intended experiment type, the mixture was kept in special containers of separate 3D printed molds for each experiment until it was fully polymerized.

The range of filling agent content in the MAE samples is based on the results of earlier research on the correlation between the MAE dielectric coefficient and the filling agent particle content, and on the authors' research on the magnetic pressure distribution (Fig. 1 a, b) [15-17].

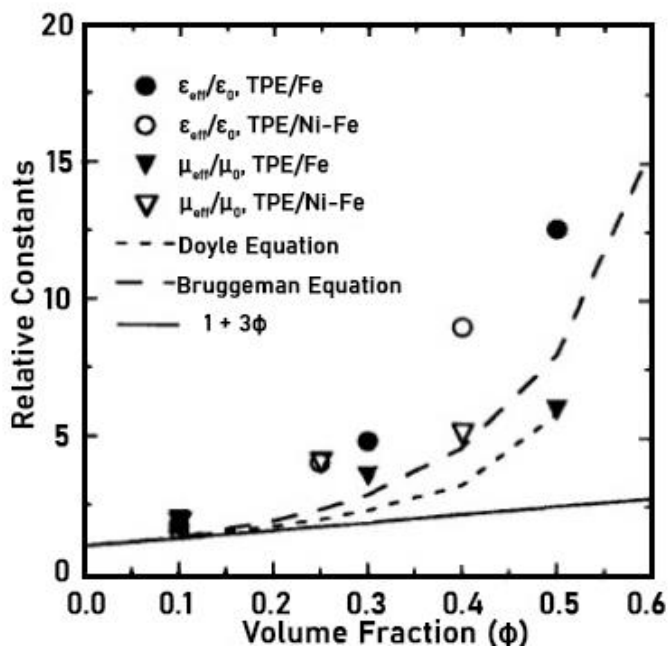

(a)

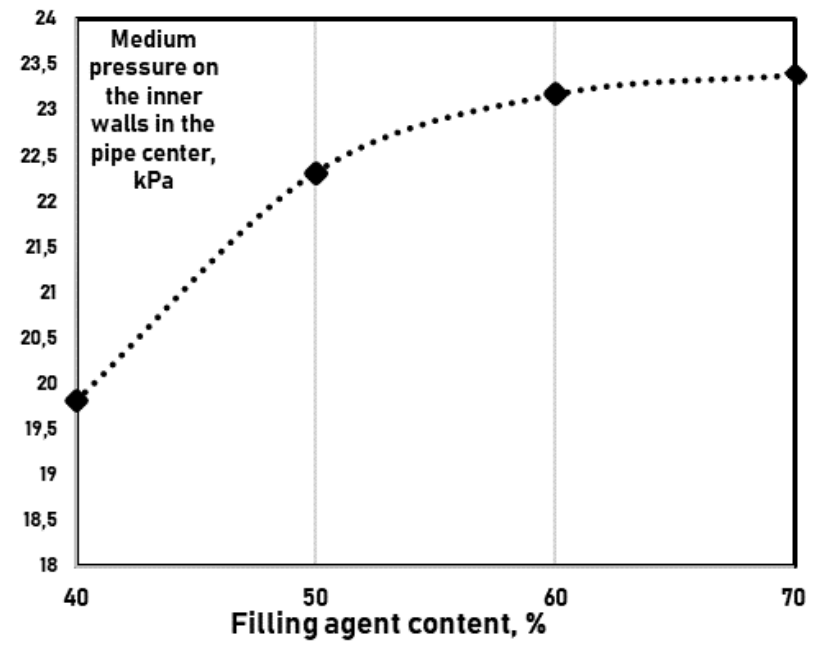

(b)

Figure 1. Magnetic properties of the material as a function of the filler content: (a) Dependence on the MAE dielectric coefficient; (b) Dependence on the magnetic pressure.

It should be noted that samples with concentrations above $70 \%$ complicate the uniform distribution of the particulate filling material in the polymerization stage due to the rapid deposition of the filler particles. Also, such samples exhibit a decrease in tensile and compressive strength of elastomers due to a decrease in bond strength at the polymer-filler junction with an increase in particle size $[18,19]$. When the concentration exceeds $80 \%$, the elastomer structure corresponds to reversed dispersion.

The samples were divided into two groups equal in number, and one of the groups was exposed to an external magnetic field of $1.2 \mathrm{~T}$ for 12 hours during the curing process. This resulted in samples with distinct structure anisotropy along the magnetic field 
lines direction (Fig. 2 c, d). The second group of samples was not subjected to further exposure in course of curing process and exhibited an isotropic structure [20 - 22].

In order to assess the mixing quality and the degree of anisotropy intensity, the research was carried out on a JSM-6460 scanning electron microscope (JEOL) in SEI and BEI mode (accelerating voltage - $20 \mathrm{kV}$, current $\mathrm{n}^{*} 10-10 \mathrm{~A}$ ) [23]. The COMPO mode was the most effective signal for the task. The samples were preliminarily fastened with carbon glue and dried for several hours under an air stream due to the material properties. The samples were pre-coated with gold for stable conductivity.

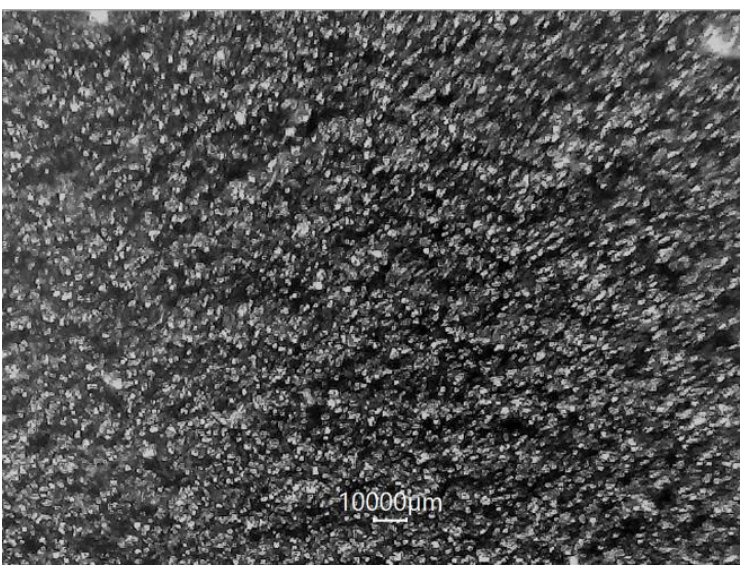

(a)

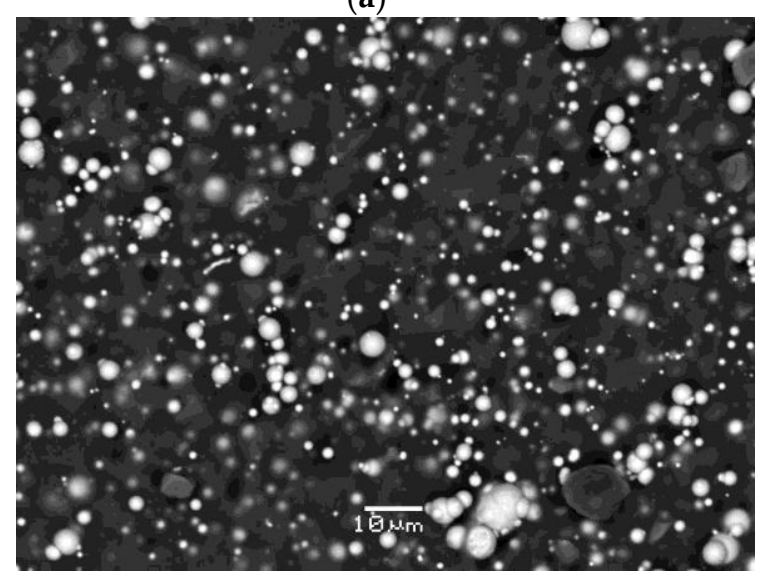

(c)

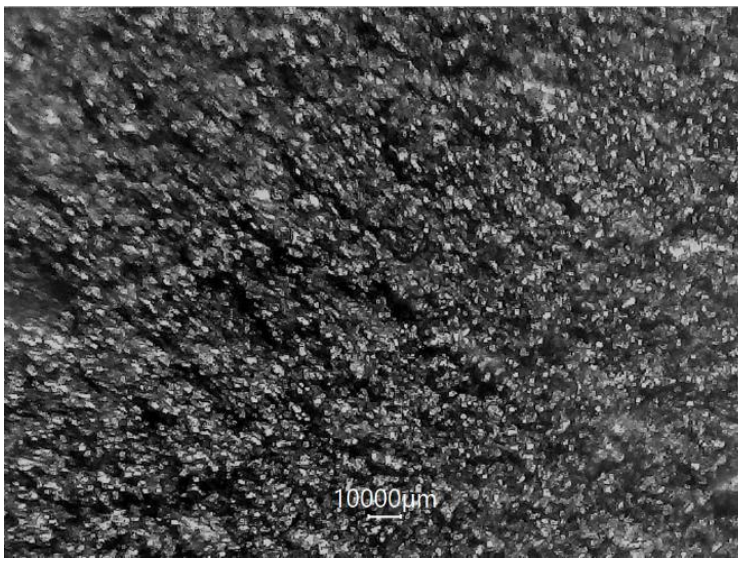

(b)

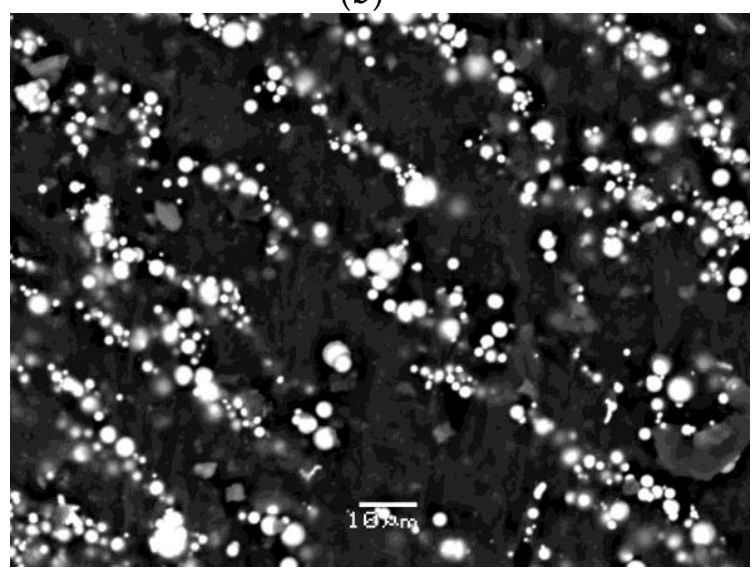

(d)

Figure 2. Filling agent particle distribution in the polymer matrix MAE*: Atomized powdered iron filler: (a) - isotropic sample, (b) - anisotropic sample; P-10 carbonyl iron filling agent: (c) - isotropic sample, (d) - anisotropic sample.

*Due to the large difference in the filling agent particle sizes, different image scales have been chosen by way of illustration.

\subsection{Magnetic field source}

In order to conduct experimental studies, samples of three different shapes were made for each set of composition and components structure. The casting molds were produced with 3D printing.

When determining the material for a pumping device, many factors must be taken into account, such as the maximum pressure, maximum loads expected in the operating channel, and the expected clearances during operation [24].

A neodymium magnet was used as the magnetic field source. The loss of magnetic properties of such magnets is not more than $2-3 \%$ over 10 years of operation (if the allowable temperature conditions are observed). The magnet is made in the shape of a $10 \times 10 \times 40 \mathrm{~mm}$ bar (Fig. 3). It is protected by a nickel coating to protect against the adverse effects of corrosion and rust. The sharp edges of the magnetic plate have been beveled. The magnetic field direction is axial. The magnet properties are shown in Table 1. 
Table 1. Main magnetic properties of $\mathrm{NdFeB}$ magnetic bar.

\begin{tabular}{cc}
\hline Parameter & Value \\
\hline Residual magnetic induction, $\mathrm{Br}$ & $1.21 \mathrm{~T}$. \\
Coercive force by magnetization, $\mathrm{Hcb}$ & $>876 \mathrm{kA} / \mathrm{m}$ \\
Coercive force by induction, $\mathrm{Hcj}$ & $>955 \mathrm{kA} / \mathrm{m}$ \\
Maximum magnetic energy, $\mathrm{BH}$ & $263-287 \mathrm{~kJ} / \mathrm{m}^{3}$ \\
Range of operating temperatures: & $-60 \ldots .+80^{\circ} \mathrm{C}$ \\
\hline
\end{tabular}

\subsection{Study of dynamic and mechanical properties of $M A E$}

The sample shape and type, along with its production method depends on the material composition, the choice was made in accordance with GOST 270-75 Rubber. Method of the determination elastic and tensile stress-strain properties. For this study, the samples were formed in double-sided blades with the appropriate set of dimensions (Fig. 3). The MAE plates of $(2.0 \pm 0.2) \mathrm{mm}$ thickness with different concentrations and material organization structure were prepared in advance, polymerized for 12 hours, and then the samples were cut out of them with beam cutters.

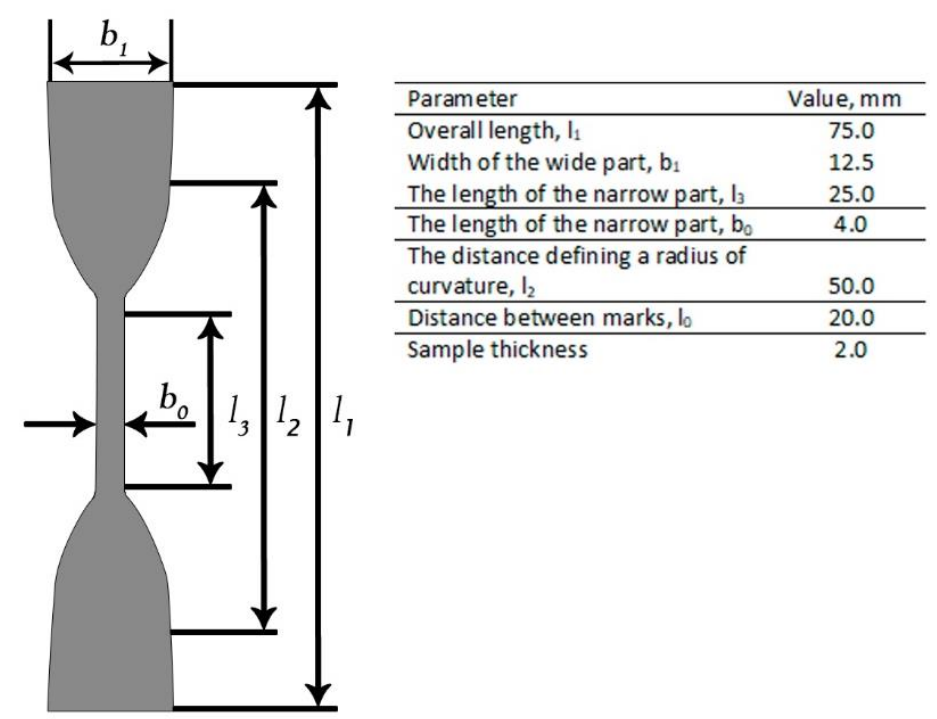

Figure 3. Test sample general view. Type III.

Except for thickness, the sample dimensions were based on cutters' dimensions and left unchecked after cutting. To ensure equal installation of the samples, installation marks were put in the grips, the distance between the marks was $(40 \pm 1) \mathrm{mm}$.

The research of the MAE stress-related properties under tension was estimated in terms of tensile strength values, elongation at rupture, and stress at a given elongation. The experimental procedures comprised three stages (Fig. 4):

a - tests at a temperature of $(23 \pm 2){ }^{\circ} \mathrm{C}$ and active grip motion velocity $-500 \mathrm{~mm} / \mathrm{min}$;

$\mathrm{b}$ - tests at temperature $(23 \pm 2){ }^{\circ} \mathrm{C}$, application of an external magnetic field with the strength of $1.2 \mathrm{~T}$ and active grip motion velocity $-500 \mathrm{~mm} / \mathrm{min}$;

$\mathrm{c}$ - tests at temperature $(70 \pm 2){ }^{\circ} \mathrm{C}$, application of an external magnetic field with the strength of $1.2 \mathrm{~T}$ and active grip motion velocity $-500 \mathrm{~mm} / \mathrm{min}$.

Studies at elevated temperature were conducted in a special thermal chamber to ensure the required temperature in the operating volume (limited by the machine traverse grips at the sample breaking point) with a tolerable error of $\pm 2.0^{\circ} \mathrm{C}$. The temperature was monitored at three points on the elastomer neck surface by the Fluke Ti450 thermal imager. 


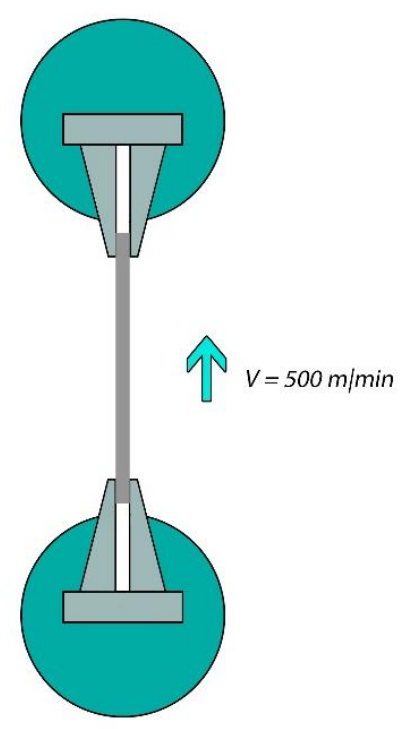

(a)

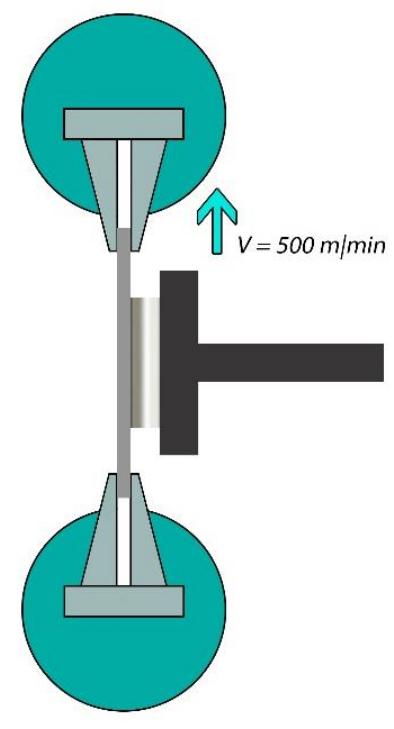

(b)

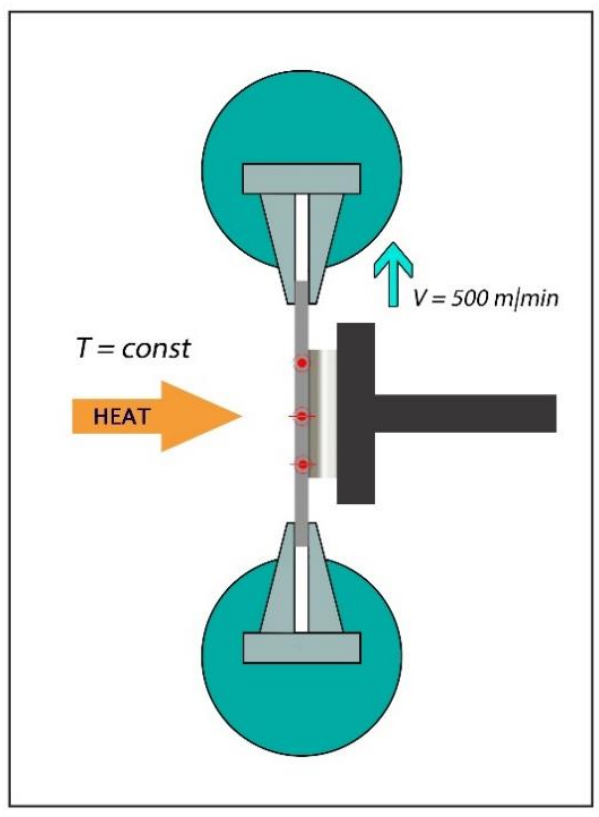

(c)

Figure 4. Diagram of the experiment to evaluate the dynamic and mechanical properties of the MAE: (a) - study without external influence; (b) - study under the influence of an external magnetic field; (c) - study under the influence of a combination of external magnetic and temperature fields.

\section{Results and discussion}

\subsection{Assessment of the material adsorption capacity}

Studies of the impact of the external magnetic field strength during the MAE polymerization at variable concentrations of the filling agent, the prepared samples with the filler content of 50, 60 and 70\% were researched by SEM analysis.

The MAE containing carbonyl iron particles has a spherical filling agent with a size of up to $5 \mu \mathrm{m}$. The samples exposed to a magnetic field exhibited a change in the distribution of these particles in the matrix relatively to a sample of similar concentration polymerized without extra exposure (Fig. 5)

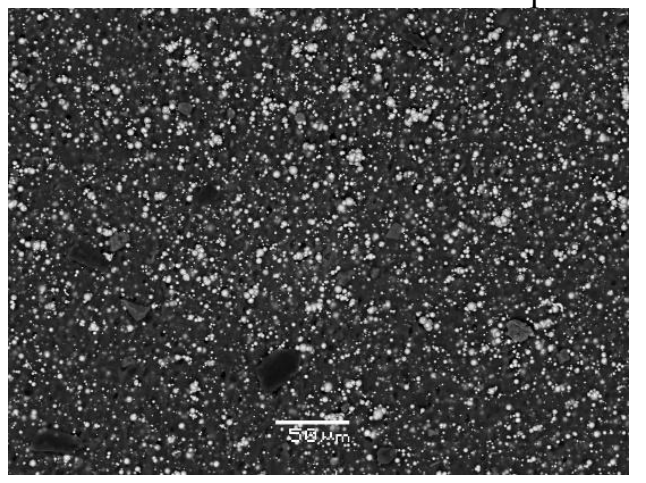

(a)

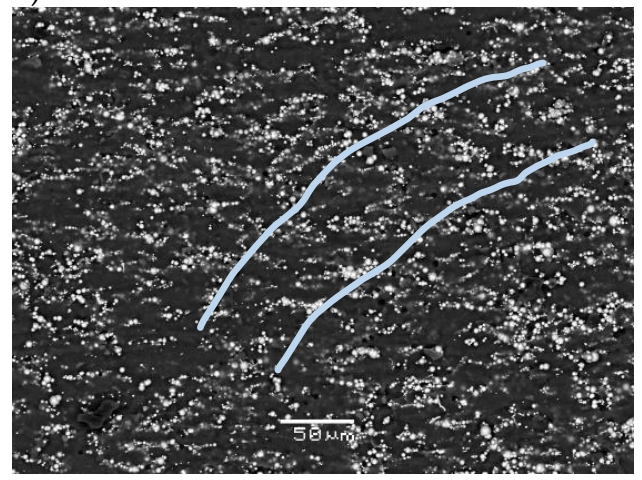

(b) 


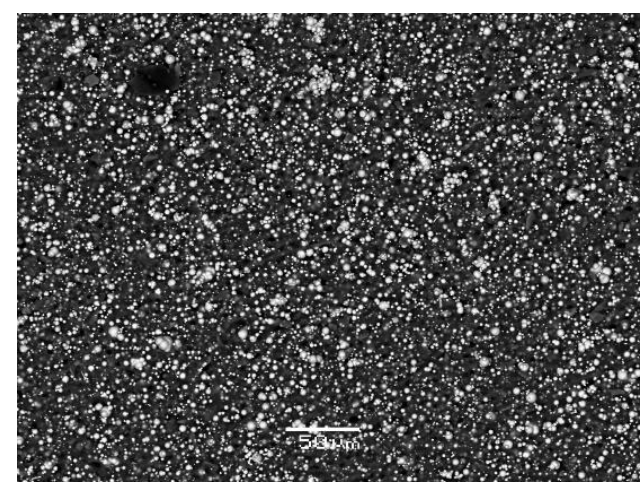

(c)

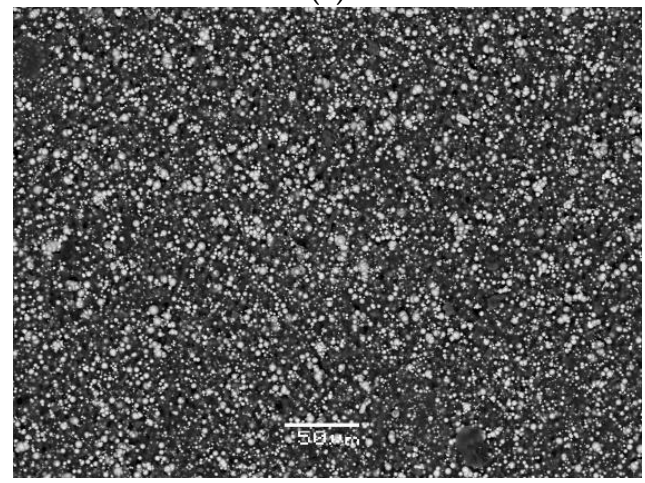

(e)

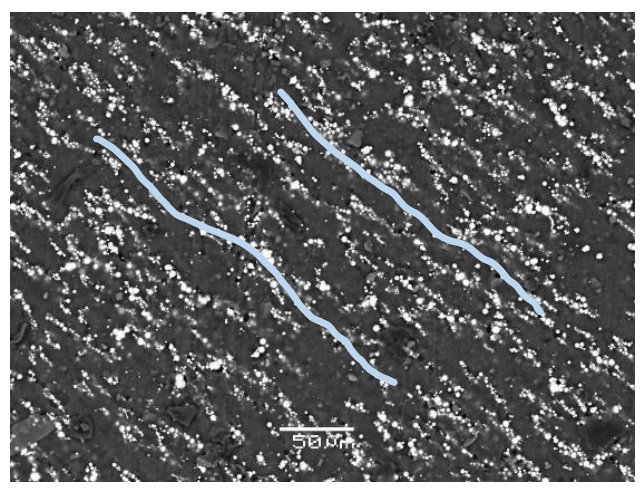

(d)

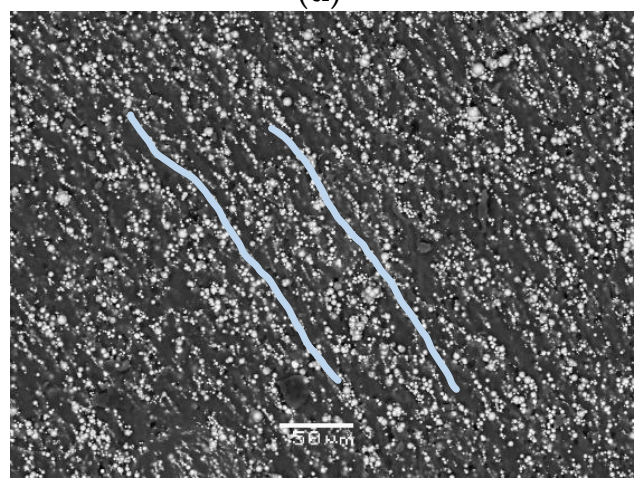

(f)

Figure 5. Internal structure of MAE samples: (a), (c), (e) - isotropic structure; (b), (d), (f) - anisotropic structure; 50, 60, 70 filling agent content, $\%$.

In the sample (b), after exposure to a magnetic field at the polymerization stage, the particles line up in elongated agglomerates parallel to the magnetic field lines; the distance between these lines is similar. The sample (d) changes its structure in a similar way. However, it is difficult to evaluate the anisotropic disposition of the particles in this sample in areas with increased agglomeration of iron particles that can range in size from $\mathrm{n}^{*} 10$ $\mu \mathrm{m}$ to $1 \mathrm{~mm}$. In the sample (e) without magnetic impact, areas of iron particles agglomeration, 100-300 $\mu \mathrm{m}$ in size, and formation of "sphere-in-sphere" structures, sized about 10 $\mu \mathrm{m}$, can also be observed. After magnetic impact, the anisotropic structure with "sphere-in-sphere" structures is denser, however, it acts differently in the agglomeration areas (Fig. 6).

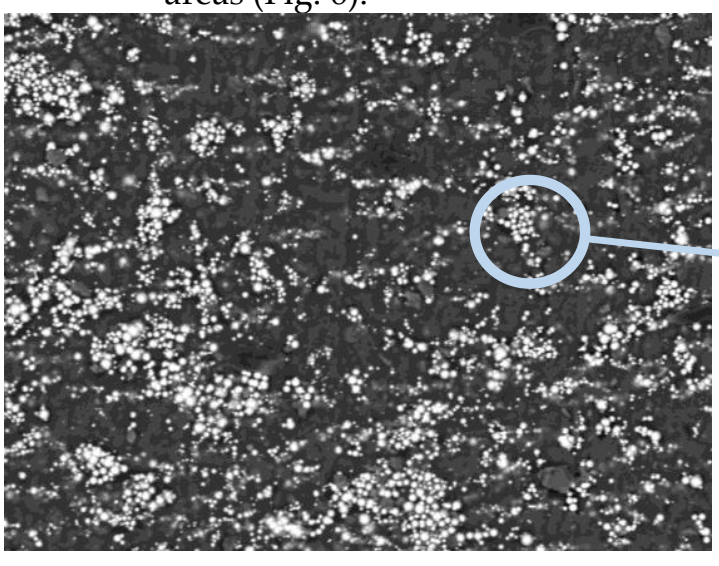

(a)

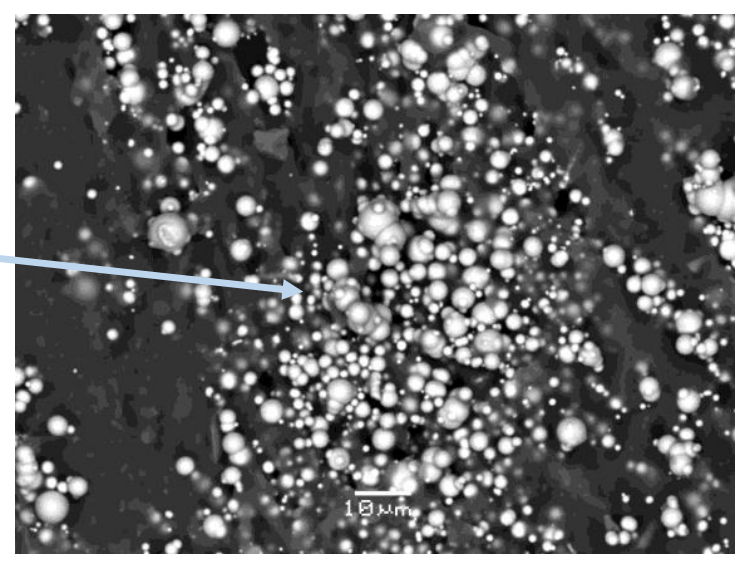

(b)

Figure 6. Agglomeration areas formation in course of MAE polymerization with P10 filler in an external magnetic field, $70 \%$ : (a) - increase up to $50 \mu \mathrm{m}(\mathrm{b})$ - increase up to $10 \mu \mathrm{m}$.

The frequency of such agglomerations significantly increases with the increase in concentration of the filling agent, it also increases their linear size. A more detailed con- 
sideration of such structures reveals an arbitrarily shaped core consisting of large particles of the filling agent [25]. Further, the smaller particles are organized in an orderly manner around the larger ones forming a spatial layered structure. This effect can be explained by the higher value of the effective magnetic field that appears as a combination of the external field and the self-induced field of large particles of the filling agent.

An increase in agglomeration ability along with a decrease in particle size and then an increase in agglomerate size is the result of the adsorption capacity [26]. The greater the tendency to agglomeration, the lower the value of the marginal volume filling omax, such tendency increases the intensity of the composite material properties change with the adsorption capacity growth.

The formation of a distinct anisotropic structure can be clearly traced in the MAE samples prepared with the use of atomized powdered iron powder as a filling agent (Fig. 7).

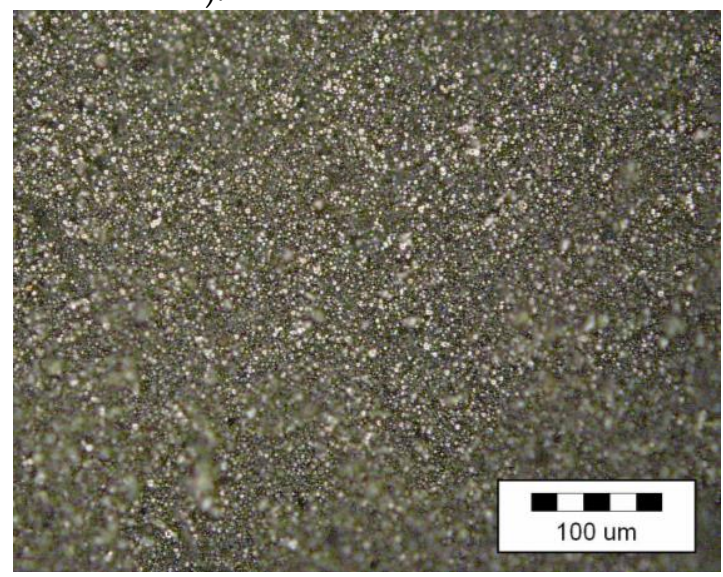

(a)

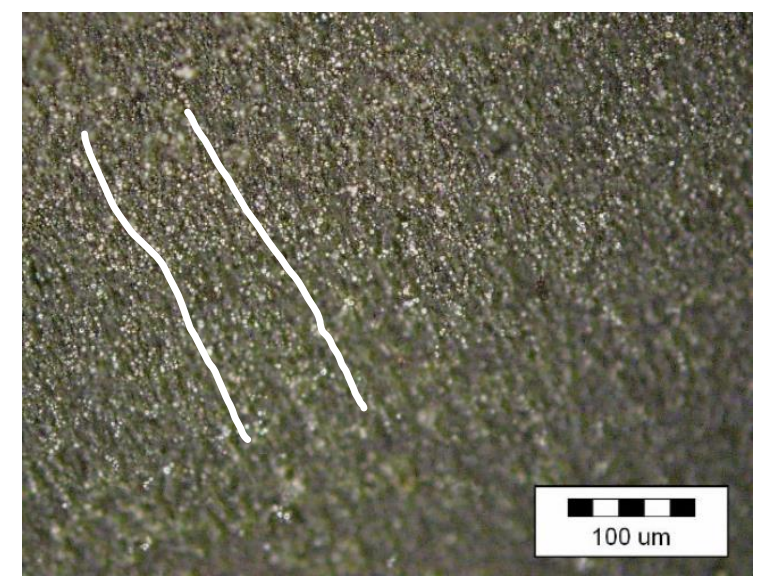

(b)

Figure 7 Internal structure of MAE samples with a filling agent content of 70\%: (a) - isotropic structure; (b) - anisotropic structure.

While the chemical composition of P10 and atomized powdered iron fillers is identical, and in spite of the MAE preparation method, the size and weight of the solid fraction particles determine the differences in the structural organization of the final material.

The filling agent distribution in the matrix material was analyzed by ToupView software, initially it allowed to create a three-dimensional representation of the image intensity, and then the final representation that corresponds to the levels of filling agent distribution in the sample was obtained by rearranging along the cut-out plane (Fig. 9). The images clearly exhibit the formation of "sphere-in-sphere" structures that in turn line up into columnar structures merging into a combined agglomerate that spreads out toward the magnetic field source. In this sector, the anisotropy is exhibited through the "sphere-in-sphere" structures lining up in the direction of the magnetic field lines.

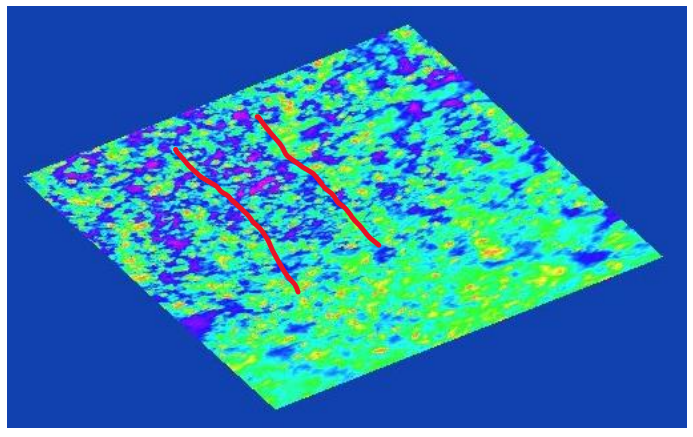

(a)

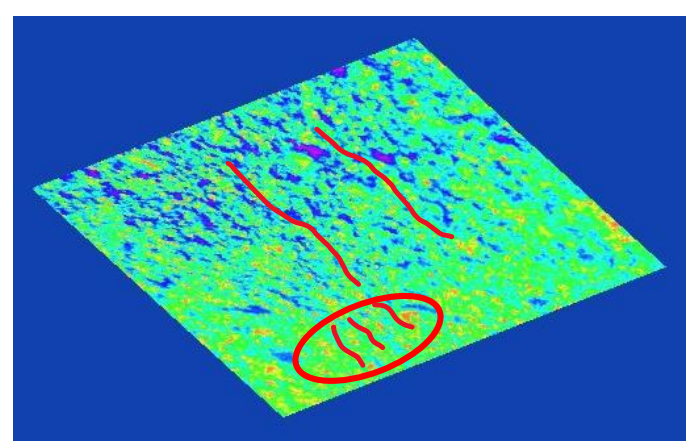

(b) 
Figure 8 Agglomeration areas formation in course of MAE polymerization with atomized powdered iron filler in an external magnetic field: (a) - $50 \%$; (b) - $70 \%$.

This kind of structural organization certainly affects the material mechanical properties resulting in a loss of stability when the content of the magnetic filling agent reaches $70 \%$ and higher (Fig. 9).

3.2 Assessment of the dynamic and mechanical properties of the material

When comparing fillers, it should be noted that the reduction in the filler grains size leads to an increase in their total specific surface area, increase in the number of particles at the same volumetric content, and, consequently, to the decrease in the distance between the filler particles in the portable optically pumped magnetometer and increase in their ability to form agglomerates.

The follow-on tests made it possible to evaluate the influence of the filling agent concentration and its structure on the main indicators of elastomers' properties with regard to their use in fluid-pumping equipment through local wave deformation: elastic modulus, MAE viscosity and strength.

By calculation, the elastic modulus of a polymer mixture can be predicted only roughly; the best match of the experimental data with the calculation can be observed in the interval of the filler concentration up to 20 and above $80 \%$; the calculations are barely suitable for the middle area of phase reversal [27].

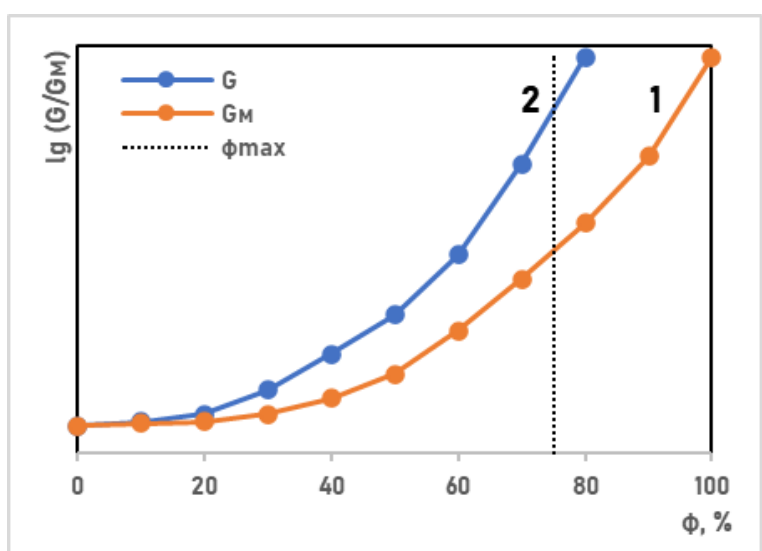

Figure 9. The dependence between the relative elastic modulus G/GM and the filling agent content $\varphi$ : 1 - design curve disregarding the limiting particle packing; 2 - curve including the limiting (dense) particle packing $\varphi_{\max }$.

The introduction of solid non-deformable filler particles leads to a decrease in the MAE plasticity along with the increase in the filler content, and the size of its particles (Fig. 10).

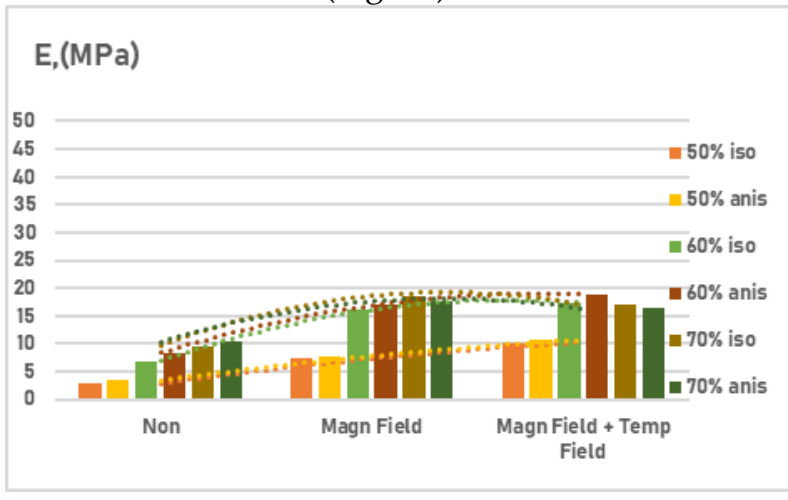

(a)

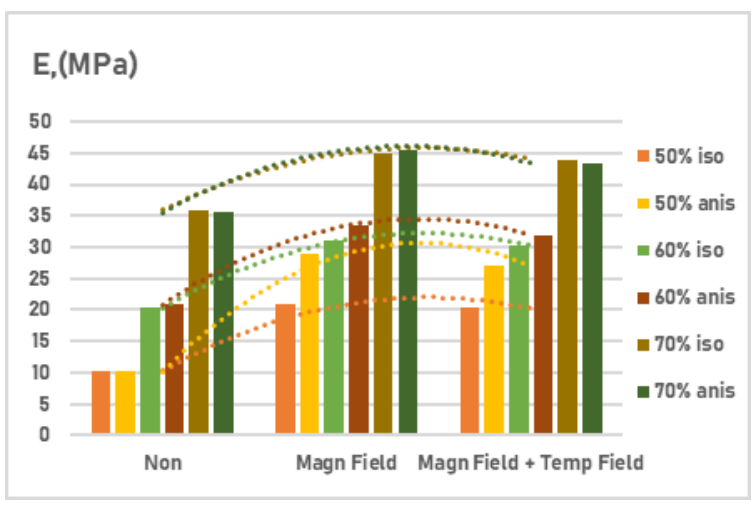

(b)

Figure 10 Agglomeration areas formation in course of MAE polymerization with atomized powdered iron filler in an external magnetic field: (a) - $50 \%$; (b) - $70 \%$. 
The development curves of the elastomers' elastic modulus indicate that MAE with atomized powdered iron filling has on average $50 \%$ higher initial elasticity compared to MAE with P10 filler. However, if we consider the samples of the same type and filler content with different structures in pairs, we notice that the anisotropic sample plasticity almost does not differ from the isotropic sample.

When evaluating the change in the material elasticity when exposed to a magnetic field, a stable increase in the material elasticity can be observed. Under the combined effect of the magnetic and temperature field, the elasticity of the material decreases slightly due to softening of the matrix material.

The value of the phase contact area in a composite elastomer is determined by the filler's specific surface area [28]. When the particle size is less than $10 \mu \mathrm{m}$, a sharp decrease in the distance between particles in the concentration range above $60 \%$ is observed.

When the material is exposed to magnetic and temperature fields, the greatest amplifying effect is recorded when the interfacial layers in the material are in contact. This condition is met when a filling agent with a larger fraction (atomized powdered iron) is applied.

Comparing the same length stretching of pure silicone and silicone-based MAE samples, it must be noted that the matrix by itself (with no solid filler involved) should provide the required deformation, and therefore it deforms harder than the pure polymer when the filler content increases $(\varphi)$ :

$$
\varepsilon_{\text {sil }}=\frac{\varepsilon_{M A E}}{1-\varphi}
$$

where $\varepsilon_{\text {sil }}$ - silicon matrix elongation.

the text following an equation need not be a new paragraph. Please punctuate equations as regular text.

Accordingly, at $50 \%$ filler particles in the MAE composition, the matrix deforms by 4 times when such a sample is elongated by 2 times, and by 6 times at 70\% [29]. As the results of experiments have shown, this inevitably leads to the MAE destruction (Fig. 11).

The composite elastomer strength correlates worse when the elastic modulus is changed and the content of the magnetoactive filling agent increases, this can be explained by the fact that the strength derives from the conditions of microdefect propagation at fracture, whereas the modulus is a property peculiar to the undamaged structure [30]. The development of shrinkage internal stresses or stress buildup on the filler particles in the deformation process greatly affects the strength and hardly affects the modulus [31].

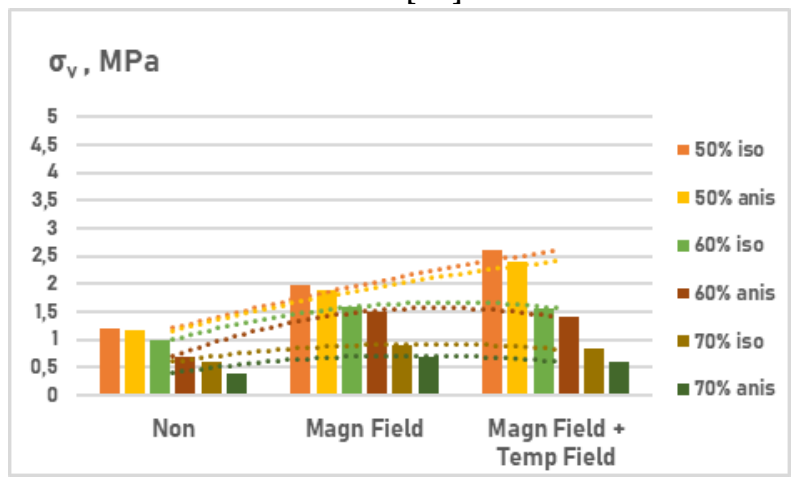

(a)

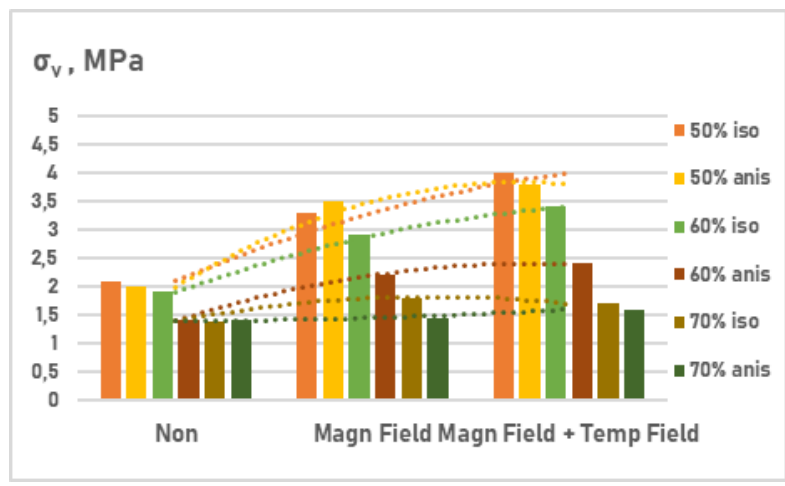

(b)

Figure 11 Research on the MAE dynamic and mechanical properties - tensile strength: (a) - samples with P10 filling agent; (b) - samples with atomized powdered iron filling agent.

The increased deformation of the matrix polymer together with the periodic stress caused by the specific operating process of the equipment along with the overstresses occurring at the matrix-filler junction will inevitably lead to microdefects in the MAE. As the relative degree of filling increases to $60-70 \%$, the overstressed areas around the parti- 
cles begin to overlap facilitating porosity growth, and the material strength decreases. This effect is caused by a reduction in the bond strength at the silicone-filling agent junction, and the strength of the material with P10 filling is lower by $38 \%$ on average because the distance between the filler particles in the MAE decreases as the number of particles per a volumetric unit at a given degree of filling increases.

This phenomenon also correlates with the Blanchard-Parkinson's theory that explains the polymer composites reinforcement through the strengthening effect caused by the formation of strong bonds 'silicone-filling agent'. Since the number of these bonds is determined by size and nature of the filler surface, the filler particles with a relatively larger contact surface area ensure good reinforcing properties.

Evaluation of the MAE strength exposed to a magnetic field, and simultaneous magnetic and temperature effects revealed that the tensile strength of anisotropic samples is significantly reduced compared to that of isotropic material. The filler particles line up in chains thus creating zones with an uneven thickness of the interfacial layer.

\section{Conclusions}

A comprehensive study of the strength properties of magnetoactive elastomers based on high permeability magnetic filling agent has made it possible to reveal the dependences of the main dynamic and mechanical properties of materials based on their composition and structure and to substantiate the composition and polymerization method of the elastomer that can be used as a material for the induction peristaltic pumping unit operating channel, namely:

1. An increase in the filler concentration in the MAE composition promotes a deformability reduction. This effect is amplified when exposed to an external magnetic field, and slightly attenuates when exposed to both magnetic and temperature fields at the same time. This attenuation effect can be explained by the faster migration of the filling agent particles, hooks and nodes of the material structure exposed to the elevated temperature.

The filling agent content above $70 \%$ leads to a decrease in the stability of the final material and is not recommended for use.

2. The anisotropic structure of the MAE leads to an enhancement of the samples' elastic properties amplified when exposed to an external magnetic field. It also contributes to the formation of agglomerates in the final material, which leads to a drop in strength properties. This trend exponentially increases as the filling agent content of the matrix increases and the grain size of the material decreases.

3. The magnetoactive elastomer prepared with a filler that has a relatively larger fraction exhibits more stable dynamic and mechanical properties - the ultimate strength of the sample rises by $38 \%$ on average, further growing with such raise; the elastic modulus is twofold higher.

4. When MAE samples are exposed to an external magnetic field, regardless of the filler size, the samples elasticity and strength increase, and this effect is particularly significant at lower concentrations; with the aggregate exposure to magnetic and temperature fields, the elasticity and strength of materials increase by $30 \%$ on average compared to a material without external influence.

5. The conducted studies indicate that MAEs with the content of filling agent about $60 \%$, with relatively larger filler fraction and isotropic structure of the final polymerized material are recommended as the most suitable material for producing equipment parts, and pumping equipment parts, in particular.

Author Contributions: Conceptualization, Vasilyeva M. and Nagornov D.; methodology, Vasilyeva M.; software, Nagornov D.; validation, Vasilyeva M., Nagornov D.; formal analysis, Nagornov D.; investigation, Nagornov D.; resources, Orlov G.; data curation, Orlov G.; writing - original draft preparation, Vasilyeva M.; writing - review and editing, Vasilyeva M.; visualization, Vasilyeva M.; supervision, Vasilyeva M.; project administration, Vasilyeva M.; funding acquisition, Vasilyeva M. All authors have read and agreed to the published version of the manuscript. 
Funding: This research was funded by RUSSIAN SCIENCE FOUNDATION, grant number 19-79-10151

\section{Institutional Review Board Statement: Not applicable.}

Informed Consent Statement: Informed consent was obtained from all subjects involved in the study.

Conflicts of Interest: The authors declare no conflict of interest. The funders had no role in the design of the study; in the collection, analyses, or interpretation of data; in the writing of the manuscript, or in the decision to publish the results.

\section{References}

1. Qiu, J. D., Lai, X. J., Fang, W. Z., Li, H. Q., Zeng, X. R. An efficient strategy for simultaneously improving tracking resistance and flame retardancy of addition-cure liquid silicone rubber. Polym. Degr. and Stab. 2017, 144, 176-186. https://doi.org/10.1016/j.polymdegradstab.2017.08.005

2. Fuhrer, R., Schumacher, C.M., Zeltner, M. and Stark, W.J., Soft Iron/Silicon Composite Tubes for Magnetic Peristaltic Pumping: Frequency-Dependent Pressure and Volume Flow. Adv. Funct. Mater. 2013, 23: 3845-3849. https://doi.org/10.1002/adfm.201203572

3. Li, Y. P., Zeng, X. R., Lai, X. J., Li, H. Q., Fang, W. Z. Effect of the platinum catalyst content on the tracking and erosion resistance of addition-cure liquid silicone rubber. Polym. Test. 2017, 63, 92-100. https://doi.org/10.1016/j.polymertesting.2017.08.017

4. Nguyen, V.Q., Ahmed, A.S. and Ramanujan, R.V. Morphing Soft Magnetic Composites. Adv. Mater. 2012, 24: 4041-4054. https://doi.org/10.1002/adma.201104994

5. Bruschi P., Ciomei A., Piotto M. Design and analysis of integrated flow sensors by means of a two-dimensional finite element model. Sens. Actuat. A, 2008, 142, 153-159

6. Lu AH, Salabas EL, Schüth F. Magnetic nanoparticles: synthesis, protection, functionalization, and application. Angew Chem Int Ed Engl. 2007, 46(8):1222-44. https://doi.org/10.1002/anie.200602866

7. Grass, RN, Athanassiou, EK, Stark WJ. Covalently functionalized cobalt nanoparticles as a platform for magnetic separations in organic synthesis. Angew Chem Int Ed Engl. 2007, 46(26): 4909-12. https://doi.org/10.1002/anie.200700613

8. Ruan, XH, Xuan, SH, Zhao, J, Bian, HT, Gong, XL. Mechanical performance of a novel magnetorheological fluid damper based on squeeze-valve bi-mode of MRF Smart Mater. and Struct., 2020, 29: 055018

9. Verte, L.A. Electromagnetic transport of liquid metal, Moscow: Metallurgy, 1965, 236 pp.

10. F. Hiptmair, Z. Major, R. Hasslacher, and S. Hild. Design and application of permanent magnet flux sources for mechanical testing of magnetoactive elastomers at variable field directions, Review of Scient. Instr. 2015, 86, 085107; https://doi.org/10.1063/1.4927714

11. Pan, Z. Q., Sun, R., Zhu, S. L., Kang, Y. Z., Huang, B. S. et al. The synthesis, characterization and properties of silicone adhesion promoters for addition-cure silicone rubber. Journal of Adhes. Scienc. and Tech, 2018, 32(14), 1517-1530. https://doi.org/10.1080/01694243.2018.1428059

12. Lu, X., Qiao, X., Watanabe, H. et al. Mechanical and structural investigation of isotropic and anisotropic thermoplastic magnetorheological elastomer composites based on poly(styrene-b-ethylene-co-butylene-b-styrene) (SEBS). Rheol Acta. 2012, 51, 37-50. https://doi.org/10.1007/s00397-011-0582-x

13. Kramarenko E, Chertovich A, Stepanov G, and others. Magnetic and viscoelastic response of elastomers with hard magnetic filler. Smart Mat. and Struct. 2015, 24, №075010

14. Tian, T., Nakano, M. Fabrication and characterization of anisotropic magnetorheological elastomer with 45 iron particle alignment at various silicone oil concentrations. Journal of Intel. Mat. Syst. and Struct., 2018, 29(2), 151-159. https://doi.org/10.1177/1045389X17704071

15. Andriushchenko P, Nefedev K, Stepanov G. Calculations of magnetoactive elastomer reactions in a uniform external magnetic field. Euro. Phys. Journal B. 2014, 87, №11

16. Kang, S.S.; Choi, K.; Nam, J.-D.; Choi, H.J. Magnetorheological Elastomers: Fabrication, Characteristics, and Applications. Materials 2020, 13, 4597. https://doi.org/10.3390/ma13204597

17. Vasilyeva, M.A., Voeth, S. A multiphysics model of the heterogeneous flow in a varying cross section duct, Journal of Mining Institute, 2017, 227. p. 558. https://doi.org/10.25515/pmi.2017.5.558

18. Boczkowska, A., Awietjan, S. F., Pietrzko, S., Kurzydłowski, K. J. Mechanical properties of magnetorheological elastomers under shear deformation. Composites Part B: Engineering 2012, 43(2), 636-640. https://doi.org/10.1016/j.compositesb.2011.08.026

19. Winger, J., Schümann, M., Kupka, A., Odenbach, S. (2019). Influence of the particle size on the magnetorheological effect of magnetorheological elastomers. Journal of Magnet. and Magn. Mater. 2019, 481, $176-182$. https://doi.org/10.1016/j.jmmm.2019.03.027

20. Kumar V, Lee D-J. Iron particle and anisotropic effects on mechanical properties of magneto-sensitive elastomers, Journal of Magnet. and Magn. Mater., 2017, 441, 105-112, https://doi.org/10.1016/i.jmmm.2017.05.049

21. Bica, I. The influence of the magnetic field on the elastic properties of anisotropic magnetorheological elastomers. Journal of Industrial and Engineering Chemistry, 2012, 18(5), 1666-1669. https://doi.org/10.1016/j.jiec.2012.03.006 
22. Lokander, M., Stenberg, B. Improving the magnetorheological effect in isotropic magnetorheological rubber materials. Polym. Test., 2003, 22, 677-680.

23. Potapov, A.I., Kondratev, A.V. Non-destructive testing of multilayer medium by the method of velocity of elastic waves hodograph, Journal of Mining Institute, 2020, 243(3), p. 348-356.

24. Ward, P., Liu, D., Waldron, K., Hasan, M. Optimal design of a magnetic adhesion for climbing robots, Nature-Inspired Mobile Robotics, 2013, 375-382.

25. Peregudin, $\mathrm{S}$, et al. The influence of dissipative effects on dynamic processes in a rotating electrically conductive liquid medium, J. Phys.: Conf. Ser. 2019, 1359, 012118

26. Andronov, G.P., Zakharova, I.B., Filimonova, N.M., L'vov, V.V., Aleksandrova, T.N., Magnetic separation of eudialyte ore under pulp pulsation, Journal of Mining Science 2016, 52(6), 1190-1194

27. Sokolova, M.D., Shadrinov, N.V., Dyakonov, A.A., Zyryanov, I.V., Application of rubber-polymer two-layer material for lining of mining equipment, Gornyi Zhurnal, 2019 (2), 66-69.

28. Zakharova, M., Uspenskaya, M.V., Podshivalov, A.A. The influence of the bentonite particles content on operating properties of gelatin/starch/glycerol/bentonite biocomposite films. 2017 International Multidisciplinary Scientific GeoConference Surveying Geology and Mining Ecology Management, SGEM, 17(51), c. 961-966

29. Dzhabbarov, S.N., Pryakhin, E.I. Research regarding heat treatment influence on properties of chromic-manganese steel with quenching in polymer solution with purpose of matching drill-stem subs. IOP Conference Series: Earth and Environmental Science 2017, 87(9), 092009

30. Potapov, A.I., Makhov, V.E. Methods for Nondestructive Testing and Diagnostics of Durability of Articles Made of Polymer Composite Materials. Russian Journal of Nondestructive Testing 2018, 54(3), 151-163

31. Vasilyeva, M.A. Justification of the choice matrix material of the magnetoactive elastomer for working camera-channel peristaltic unit, Material Science Forum 2016, 870, 13-19. 